\title{
MITRAL ATRESIA ASSOCIATED WITH PULMONARY VENOUS ANOMALIES*
}

\author{
BY \\ JOHN D. SHONE AND JESSE E. EDWARDS \\ From the Departments of Pediatrics and Pathology, University of Minnesota, and the Department of Pathology, The \\ Charles T. Miller Hospital, St. Paul, Minnesota, U.S.A.
}

Received July 1, 1963

In mitral atresia with a closed or narrowly patent foramen ovale, anomalous pulmonary venous connexions may occur that function as collateral pathways for the return of pulmonary venous blood to the heart. In 2 cases (Edwards and DuShane, 1950; Lucas et al., 1962), although all the pulmonary veins connected normally, an anomalous channel-termed a lævo-atriocardinal veinformed the collateral pathway connecting the left atrium to the left innominate vein.

This report describes coexisting pulmonary venous anomalies in 5 cases among a group of 29 cases of mitral atresia studied by us. In 4 of these 5 , the anomalous pulmonary veins took the form of anomalous pulmonary venous connexion: in the remaining 1 the pulmonary venous anomaly was represented by cor triatriatum. In 3 of the 5 cases (Group I) mitral atresia and premature closure of the foramen ovale coexisted. The anomalous pulmonary venous connexions in this group provided collateral routes for the flow of pulmonary venous blood, and can be understood as developing in response to obstruction at the foramen ovale when the mitral valve is atretic.

In the remaining 2 cases (Group II) no such causative factor could be invoked for the presence of the pulmonary venous anomalies, since in each an interatrial communication was present. In one of the latter group total anomalous pulmonary venous connexion coexisted with a common atrium. In the other, cor triatriatum coexisted with a patent foramen ovale-the four individual pulmonary veins connecting to the upper (accessory) left atrial chamber.

In all 5 cases, regardless of the specific anatomical arrangement, obstruction to pulmonary venous flow occurred. Grossly, in the 4 cases where the lungs were available for study, this organ appeared much congested and œdematous and the pleural surfaces showed a reticulated pattern of distended lymphatic channels. Histologically, there was medial hypertrophy of muscular arteries with very prominent elastic membranes, medial hypertrophy of the arterioles, congestion of alveolar walls, and dilatation of lymphatics (Fig. 1).

The purpose of this report is to define the pulmonary venous anomalies in 4 of the $5 \dagger$ cases. Two of them (Cases 1 and 3) indicate the complexity of the pulmonary venous system that may develop when obstruction to flow is present during the stages when the lungs and their respective vascular beds are developing. The material also will indicate how normal mediastinal structures may contribute to obstruction of anomalous pulmonary veins.

* This study was supported by Research grant HE-5694 of the National Heart Institute, United States Public Health Service.

$\dagger$ One of the 5 cases, an example with a lævo-atriocardinal vein, has been reported by Lucas et al. (1962). In this case-that of an 11-week-old female infant-mitral atresia coexisted with partial premature closure of the foramen ovale. Total anomalous pulmonary venous drainage occurred through a lævo-atriocardinal vein to the left innominate vein. Also present were a ventricular septal defect, hypoplastic left ventricle, tubular hypoplasia of the aortic arch, and a patent ductus arteriosus. 

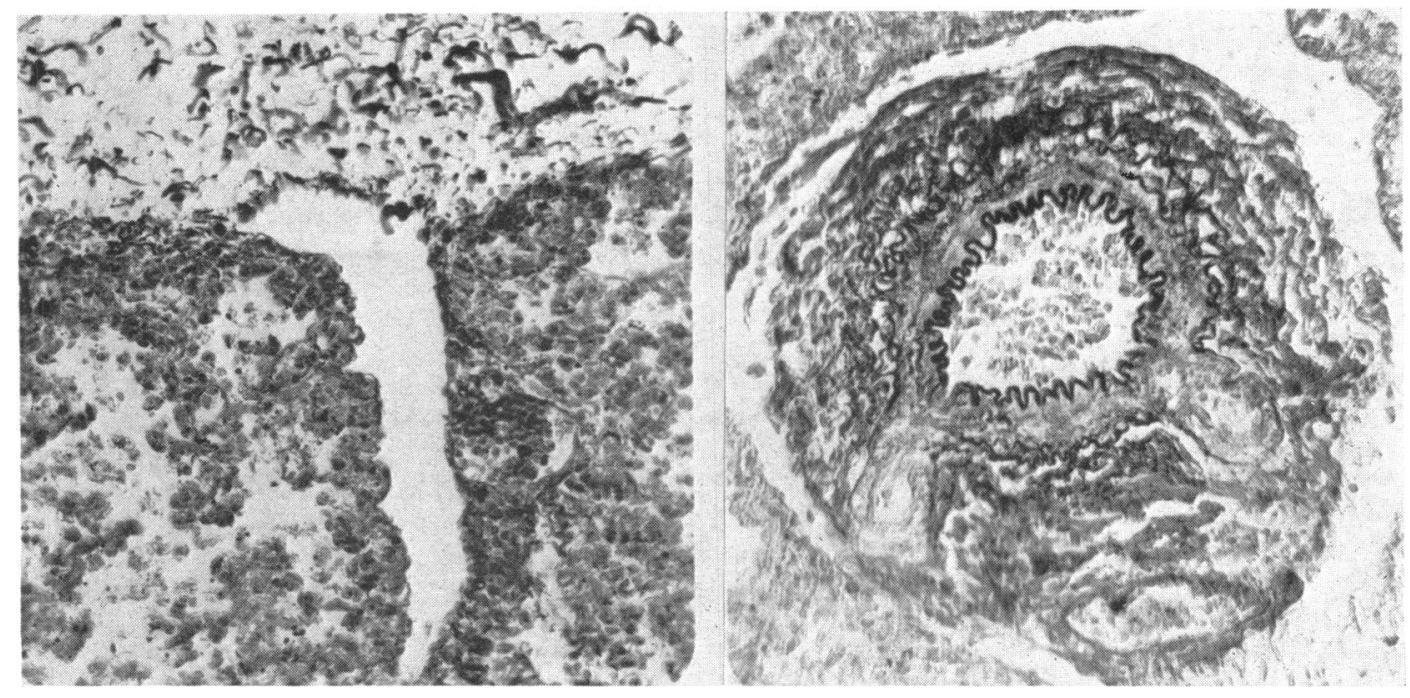

FIG. 1.-Case 3. Photomicrographs of pulmonary vessels. The histological changes (considered to have resulted from pulmonary venous obstruction) are representative of the 5 cases reported. Left: engorgement of alveolar capillaries and a dilated lymphatic channel. (H. and E. $\times 250$.) Right: muscular artery showing prominent elastic membranes and medial hypertrophy. (Elastic tissue stain. $\times 200$.)

\section{Group I: Mitral Atresia with Premature Closure of Foramen Ovale}

Case 1. Mitral atresia; premature closure of the foramen ovale; anomalous left upper pulmonary vein; interlobar pulmonary venous connexions. Also coarctation of the aorta, patent ductus arteriosus, hypoplastic left ventricle, and a ventricular septal defect were present.

In a newborn male infant with normally interrelated great arteries, the mitral valve was atretic, and the foramen ovale prematurely closed. The endocardium of the small left atrium was thickened. The upper and lower right pulmonary veins and the lower left pulmonary vein joined the left atrium at normal sites (Fig. 2), while the left upper vein did not join the left atrium. In the interlobar fissure venous radicles of the left lower pulmonary lobe passed into the substance of the left upper pulmonary lobe (Fig. 4). From the intrapulmonary concourse of these radicles the anomalous left upper pulmonary vein was formed. After leaving the medial aspect of the upper lobe of the left lung, the anomalous left upper pulmonary vein passed medially, inferior to the left pulmonary artery (Fig. 3, left). It then turned abruptly posteriorly, passing between the left pulmonary artery antero-superiorly and the left main bronchus postero-inferiorly. In the latter position the vein was compressed by these contiguous structures (Fig. 3 and 5). The left upper pulmonary vein then turned vertically, and, while its termination was not identified, its position and course were consistent with a termination in the left innominate vein (Fig. 5). Inferior to its compression by the left pulmonary artery and bronchus the left upper pulmonary vein measured $3 \mathrm{~mm}$. in diameter. Above this level it measured $1 \mathrm{~mm}$. in diameter.

In the presence of coexistent mitral atresia and premature closure of the foramen ovale, it is obvious that the direction of pulmonary venous blood flow was abnormal. Reference to the anatomical arrangement of the pulmonary veins (Fig. 5) provides an explanation for the route taken by the pulmonary venous blood. From the right lung the venous blood entered and crossed the left atrium to flow retrogradely into the left lower pulmonary vein. From this latter vein, blood of the right lung and of the lower lobe of the left lung proceeded superiorly through the interlobar anomalous venous channels into the left upper pulmonary vein, where it was joined by blood from the upper lobe of the left lung. The entire pulmonary venous flow then proceeded superiorly in the anomalous left upper pulmonary vein. It is presumed from the anatomical details available to us that the pulmonary venous blood was then carried to the left innominate vein for delivery to the right atrium.

Pulmonary venous obstruction on the basis of the mitral atresia and premature closure of the foramen ovale was augmented by compression of the anomalous left upper pulmonary vein (which carried all the 


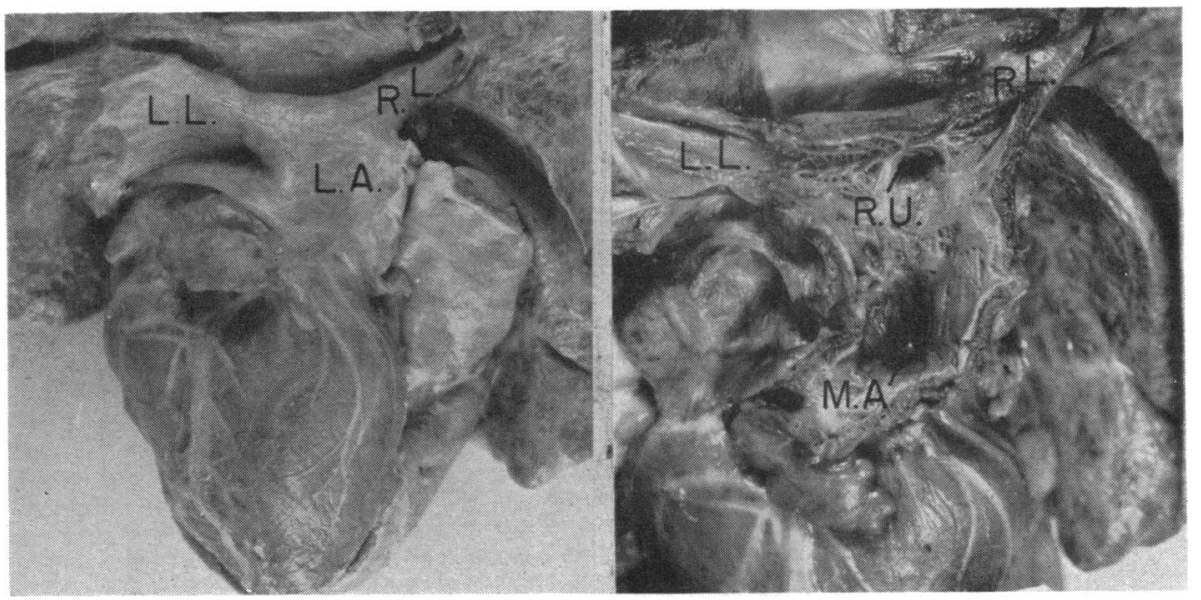

FIG. 2.-Case 1. Left: posterior aspect of exterior of heart. The right lower (R.L.) and left lower (L.L.) pulmonary veins join the left atrium (L.A.), as does the right upper pulmonary vein (latter not in view). Right: interior of left atrium. The right upper (R.U.), right lower (R.L.) and left lower (L.L.) pulmonary veins join the left atrium at normal sites. No ostium for the left upper pulmonary vein in the left atrium. A blind-ending dimple marks the site of the atretic mitral valve (M.A.).
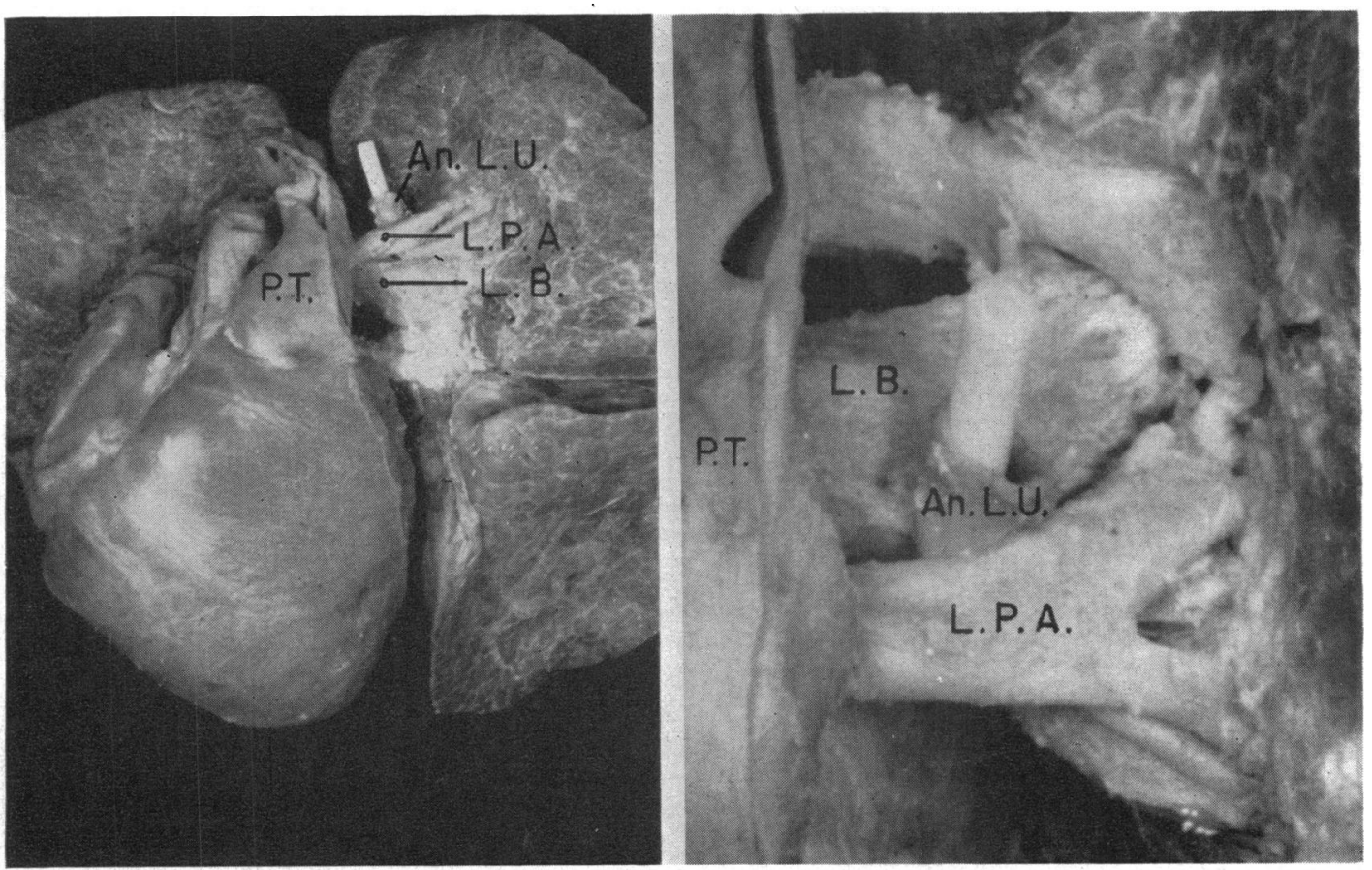

FIG. 3.-Case 1. Anterior aspect of the heart and lungs. Left: the anomalous left upper pulmonary vein (An. L.U. (containing probe) ascends behind the left upper pulmonary artery (L.P.A.). P.T.=pulmonary trunk; L.B. = left main bronchus. Right: the hilar area of the left lung viewed from above and slightly in front. The anomalous left upper pulmonary vein (An. L.U.) ascends posterior to the left pulmonary artery (L.P.A.) and anterior to the left main bronchus (L.B.). Within the confined space between the artery and the bronchus the anomalous vein is compressed. 


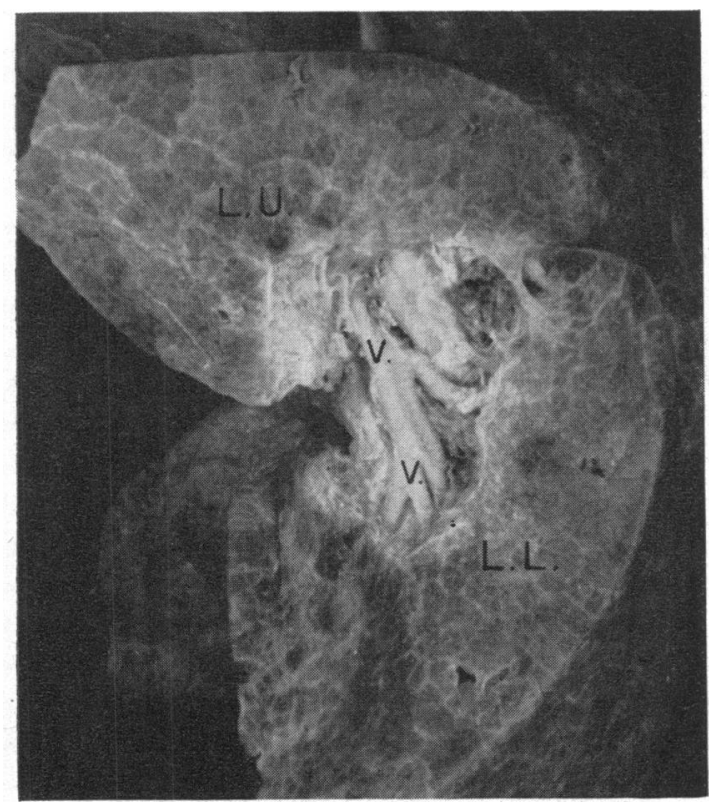

FIG. 4.-Case 1. Lateral aspect of the left lung. The left upper (L.U.) and left lower (L.L.) pulmonary lobes have been displaced apart exposing the interlobar fissure. In the interlobar fissure venous radicles (V.) from the left lower pulmonary lobe (L.L.) pass into the left upper pulmonary lobe (L.U.). Reticulated pattern on pulmonary surfaces represents dilated lymphatics.

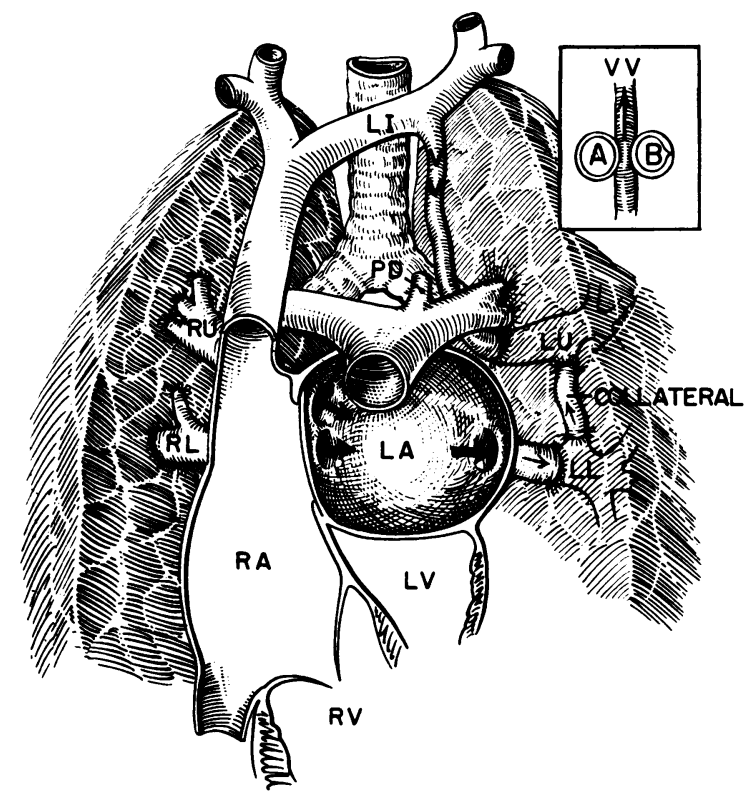

FIG. 5.-Diagrammatic representation of the anomalies in Case 1. In the presence of mitral atresia and a closed foramen ovale, blood from the right upper (R.U.) and right lower (R.L.) pulmonary veins crosses the left atrium (L.A.) and flows (in the direction indicated by the arrows) retrogradely into the left lower (L.L.) pulmonary vein. Blood from the left lower pulmonary vein then flows through collateral channels in the left interlobar fissure (Fig. 4) to join the anomalous left upper pulmonary vein. The latter (V.V.) joins the left innominate vein (L.I.). Insert. The anomalous left upper pulmonary vein (V.V.) is compressed in its vertical course by the left pulmonary artery (A) anteriorly and by the left main bronchus (B) posteriorly. R.A. = right atrium; R.V.=right ventricle; L.V.=left ventricle; P.D. = patent ductus arteriosus.

pulmonary venous blood) as it coursed between the left pulmonary artery and the left main bronchus. This anatomical phenomenon of compression of an anomalous pulmonary vein by contiguous structures is comparable to that described by Kauffman, Ores, and Andersen (1962) and by Elliott and Edwards (1962). The latter authors referred to this phenomenon of compression as a "hæmodynamic vice."

Case 2. Mitral atresia; premature closure of the foramen ovale; hypoplasia of the right upper pulmonary vein. Hypoplastic left ventricle and a ventricular septal defect also were present.

In a newborn infant (sex unknown) with normally interrelated great arteries, the mitral valve was atretic, and the foramen ovale prematurely closed. In the necropsy specimen available for examination the pulmonary veins had been excised near the left atrium and the lungs had not been submitted for study. In the wall of the left atrium, the ostia of the upper and lower right pulmonary veins were present at normal sites (Fig. 6). The right upper pulmonary vein was, however, hypoplastic at its veno-atrial junction, its external diameter being about $2 \mathrm{~mm}$. On the left side a single wide venous trunk joined the left atrium.

No egress of blood from the left atrium was possible through the atretic mitral valve or through the closed foramen ovale. The single wide venous trunk that joined the left atrium on the left side may have been formed by the confluence of the upper and lower left pulmonary veins. On the other hand, it may have represented a wide left lower pulmonary vein (distended by retrograde flow from the left atrium) which, as in Case 1, might have connected with an anomalous left upper pulmonary vein terminating in the left inno- 


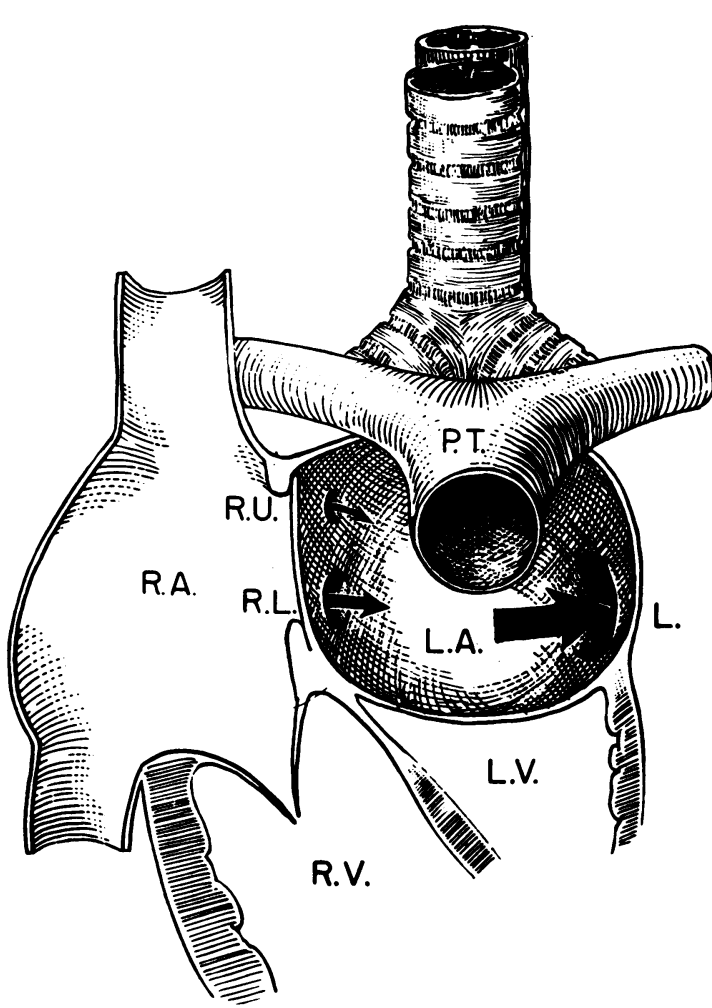

FIG. 6.-Diagrammatic representation of the anomalies in Case 2. The ostia of the right upper (R.U.) and right lower (R.L.) pulmonary veins of the left atrium are at normal sites. The right upper pulmonary vein is hypoplastic at its veno-atrial junction. On the left side, a single wide venous trunk (L.) joins the left atrium. In the presence of mitral atresia and a closed foramen ovale, it is assumed that blood flowed in the direction indicated by the arrows (comparable to the arrangement in Case 1). Because of the deficiencies in the specimen this point could not be established. It is assumed, however, that anomalous connexion from a left pulmonary vein to a systemic venous site had existed as a collateral channel. R.A. = right atrium; L.A. = left atrium; R.V.= right ventricle; L.V.=left ventricle; P.T.=pulmonary trunk.

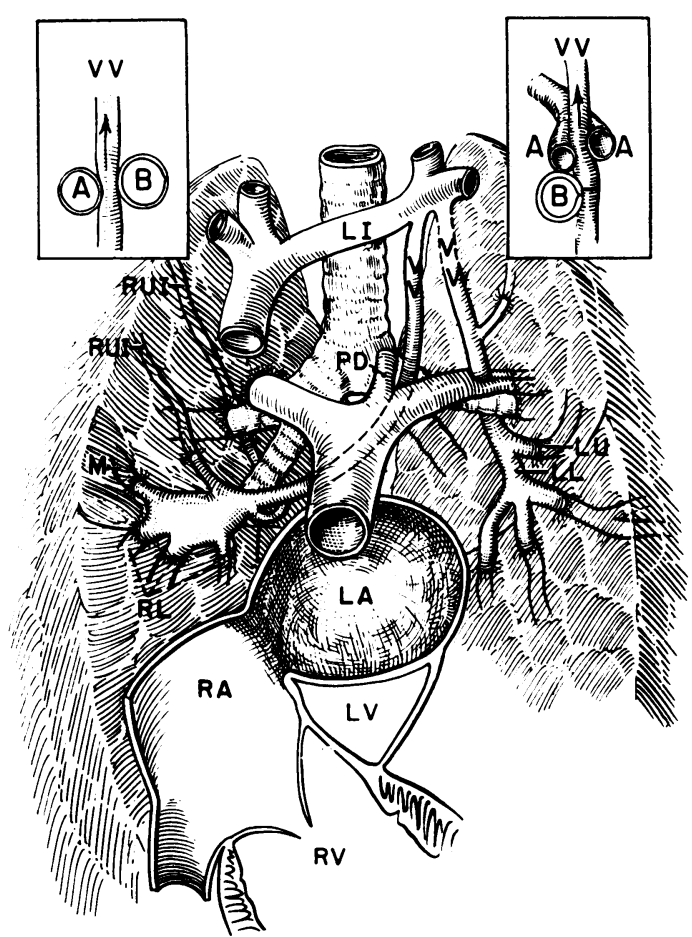

FIG. 7.-Diagrammatic representation of anomalies in Case 3. Mitral atresia is associated with absence of the atrial septum. None of the pulmonary veins connect directly with the common atrium (RALA). The pulmonary veins from the right lung (RUI, M, and RL) converge to form a single venous channel which crosses the midline to terminate in the left innominate vein. During its ascent this anomalous vein (VV) is compressed between the left pulmonary artery (A) anteriorly, the left main bronchus (B) posteriorly (left insert), and by the patent ductus (PD) medially. Venous radicles from the left lower lobe (LL) join with radicles from the left upper lobe (LU) to form a second anomalous vertical pulmonary vein (VV) which ascends posterior to the left main bronchus. During its ascent this vein is compressed between two branches $(\mathrm{A}, \mathrm{A})$ of the left pulmonary artery (right insert).

minate vein. The narrowing of the right upper pulmonary vein indicates that it was an inadequate channel. One must consider that blood in the bed of the right upper pulmonary vein might have been carried through intrapulmonary venous collaterals to the right lower pulmonary vein. Such a phenomenon was present in a case of focal pulmonary venous stenosis reported by Becu et al. (1955).

\section{Group II. Mitral Atresia with Interatrial Communication}

Case 3. Coexistent mitral and aortic valvular atresia; absence of atrial septum; total anomalous pulmonary venous connexion to left innominate vein. Hypoplasia of the left ventricle and ascending aorta, and a patent ductus arteriosus also were present.

In a newborn infant (sex unknown) with normally interrelated great arteries, mitral and aortic valvular atresia coexisted. None of the pulmonary veins connected directly with the common atrial chamber. The 


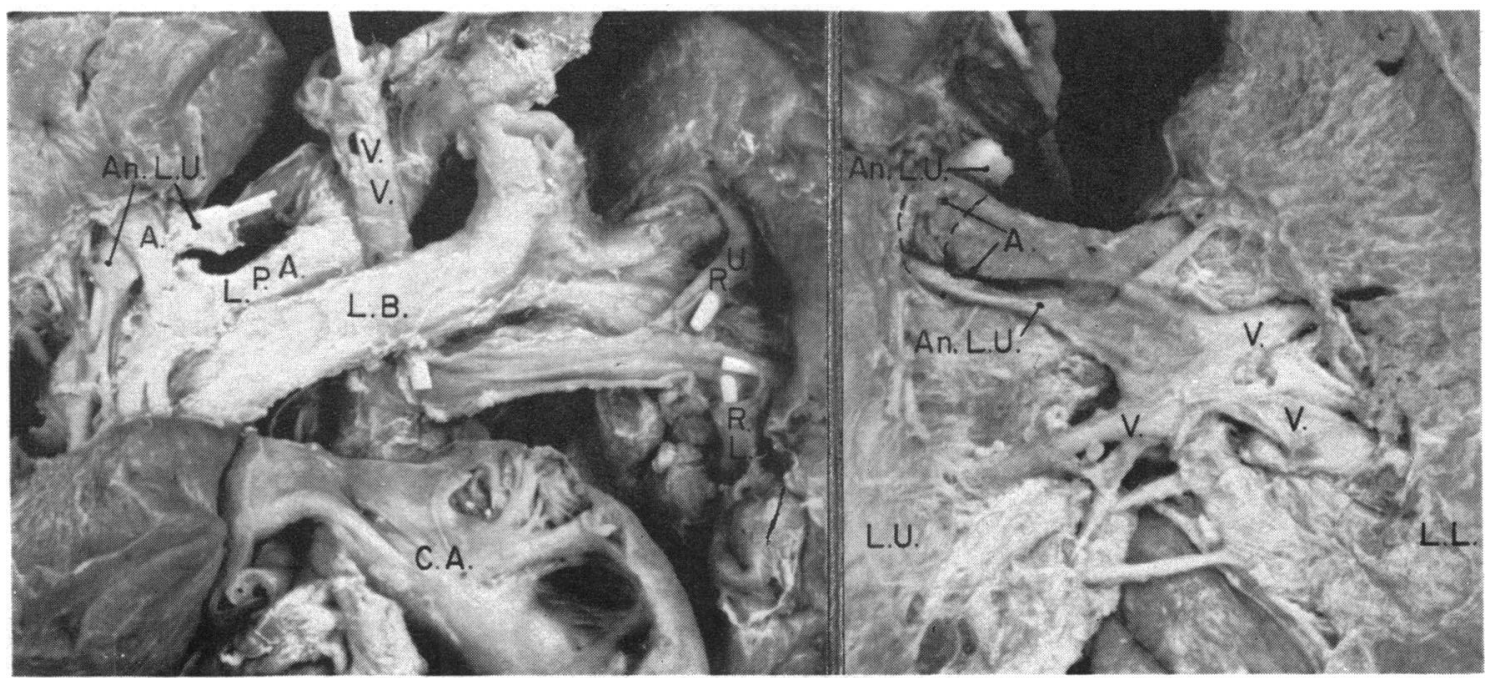

FIG. 8.-Case 3. Left: tracheal bifurcation, hilar regions of lungs, and common atrium (C.A.) from behind. None of the pulmonary veins connects with the common atrium (C.A.). The right upper (R.U.) and right lower (R.L.) pulmonary veins converge to form a single venous channel, which crosses the midline, and then ascends (V.V.) anterior to the left main bronchus (L.B.) and posterior to the left pulmonary artery (L.P.A.). The anomalous left upper pulmonary vein (An. L.U.) ascends posterior to the left upper lobe bronchus before passing through an arterial fork formed by two branches of the left pulmonary artery (L.P.A.). Only one of these branches (A.) is seen in this perspective. (This relation is shown diagrammatically in right insert of Fig. 7.) Right: left lung viewed in interlobar aspect. The left upper (L.U.) and left lower (L.L.) pulmonary lobes have been displaced apart to expose the interlobar fissure. In the interlobar fissure venous radicles (V.) from the lower lobe (L.L.) connect with those from the upper lobe (L.U.). From the concourse of these radicles an anomalous left upper pulmonary vein (An. L.U.) is formed which passes between 2 branches of an artery (A.) to the left upper lobe (points of arrows) and posterior to the left upper lobe bronchus (see right insert of Fig. 7). (The course of the anomalous left upper pulmonary vein between these 2 arterial branches is indicated by broken lines.)

upper and lower right pulmonary veins converged to form a single venous channel that crossed the midline and then coursed upwards to terminate in the left innominate vein (Fig. 7 and 8, left). During its ascending course, this anomalous vein was compressed by the left pulmonary artery anteriorly, by the left main bronchus posteriorly (Fig. 7, left insert), and by the patent ductus arteriosus medially. The veins from the left lung also showed unusual features. In the left interlobar fissure, venous radicles from the lower lobe connected with those from the upper lobe (Fig. 8, right).

From the concourse of these radicles an anomalous left upper pulmonary vein was formed. This vein ascended posterior to the left upper bronchus. It then passed through a narrow fork formed by two branches of the artery to the upper lobe of the left lung (Fig. 7 and 8). Two sites of compression of this vein were identified. One was in the aforementioned arterial fork; the other was below this level. At the latter site the bronchus (supplying the upper lobe of the left lung) pressed on the anterior wall of the vein as it was held fixed in the arterial fork above (Fig. 7, right insert). While the site of termination of the anomalous left upper pulmonary vein could not be identified, its course and position indicated that, like the anomalous vein from the right lung, it had joined the left innominate vein.

The total anomalous pulmonary venous connexion in this case was interesting from several points of view. It is uncommon to discover two separate sites of anomalous termination of pulmonary veins. When this does occur the two sites of termination are usually remote from each other (Burroughs and Edwards, 1960). In the case reported here the anomalous vein from the right lung entered the left innominate vein, and the course and position of the anomalous left pulmonary vein suggested that the latter also joined the left innominate vein.

In mitral atresia the phenomenon of pulmonary venous obstruction is usually dependent, wholly or in part, on an inadequate interatrial communication for the exit of blood from the left atrium. In this case, the atrial septum was absent allowing free communication between the atria. The pul- 


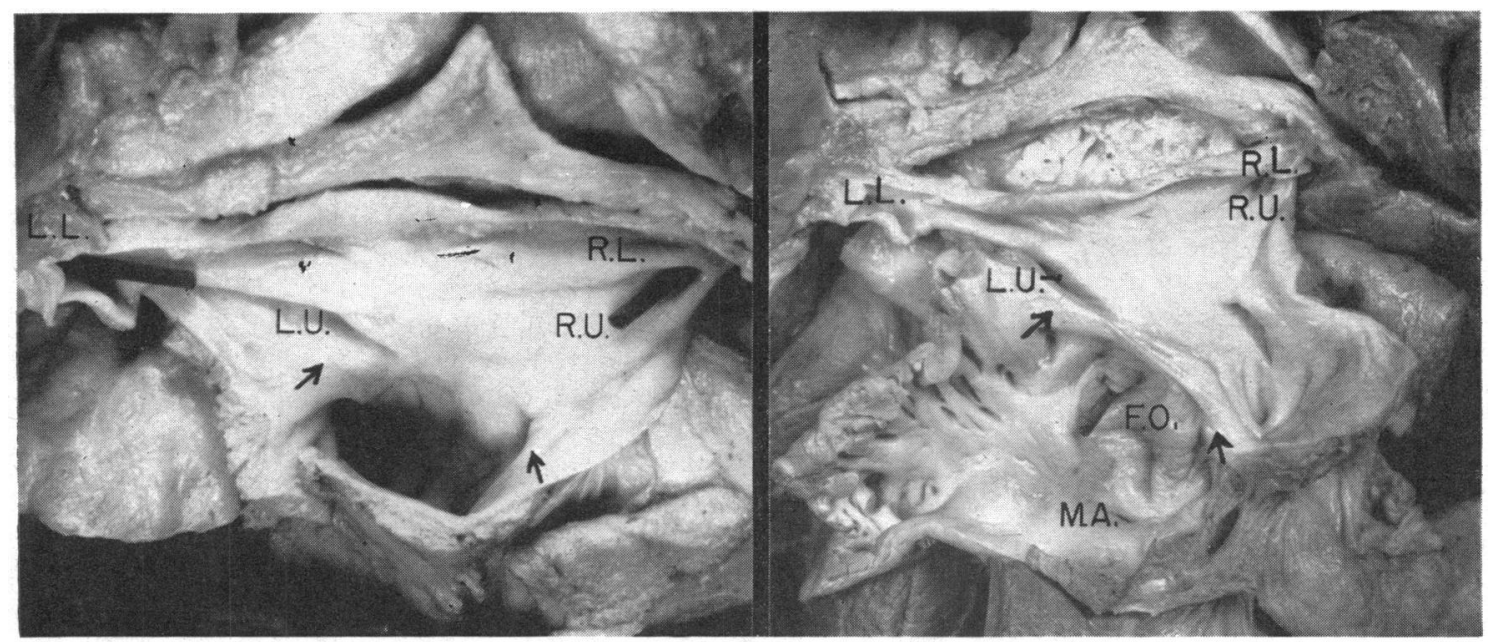

FIG. 9.-Case 4. Left: cor triatriatum. Pulmonary veins and interior of accessory (left) atrial chamber viewed from postero-superior aspect. The four individual pulmonary veins (L.L., L.U., R.L., R.U.) join the accessory atrial chamber. This chamber communicates through a relatively wide orifice in its floor (arrows) with the true left atrium below. Right: the lower (true) left atrial cavity has been opened. The ridge of tissue between the accessory and true left atrial chambers lies between the arrows. The atretic mitral valve (M.A.) is represented by a blind-ending dimple. F.O.= foramen ovale.

monary venous obstruction resulted from compression by mediastinal structures of the anomalous pulmonary veins.

In Case 1, the demonstrated, and in Case 2, the assumed, anomalous connexion of pulmonary veins could be explained on the basis that there were collateral channels in the face of obstruction to blood flow from the left atrium. No such obstructive factor existed in this case to explain why the pulmonary veins connected anomalously.

Case 4. Mitral atresia; cor triatriatum; patent foramen ovale. Also present were two ventricular septal defects, a patent ductus arteriosus, a bicuspid aortic valve, tubular hypoplasia of the aortic arch, hypoplasia of the left ventricle, atresia of the right atrial ostium of the coronary sinus, and anomalous origin of the right subclavian artery.

In a 2-year-old girl, normally interrelated great arteries, mitral atresia, and a patent foramen ovale coexisted with cor triatriatum. The four individual pulmonary veins joined a well-defined, accessory left atrial chamber. This chamber communicated through a relatively wide orifice in its floor with the true left atrium below (Fig. 9). The lower chamber communicated with the left atrial appendage and, through a narrow patent foramen ovale, with the right atrium (Fig. 10). The atrial wall in the region of the foramen ovale bulged into the right atrium. Elevated pressure in the true left atrium caused by the mitral atresia produced the bulging of the atrial septal wall into the right atrium, and this, in turn, produced patency of the foramen ovale from posterior displacement of the valve of the foramen ovale. The relatively wide orifice between the accessory and true left atrial chambers would provide little, if any, obstruction to pulmonary venous blood flow. It is considered, therefore, that pulmonary venous obstruction in this case occurred on the basis of the coexistent mitral atresia and narrow patency of the foramen ovale.

No collateral pathways for the return of pulmonary venous blood were present. The cor triatriatum is considered, therefore, to have developed at or about the same time as the development of the mitral atresia, and to be coincidentally, not causally, related.

\section{COMMENT}

In early stages of pulmonary development the primordia of both lungs and their respective lobes are represented by one mass of tissue with a common vascular bed. As the two lungs are formed 


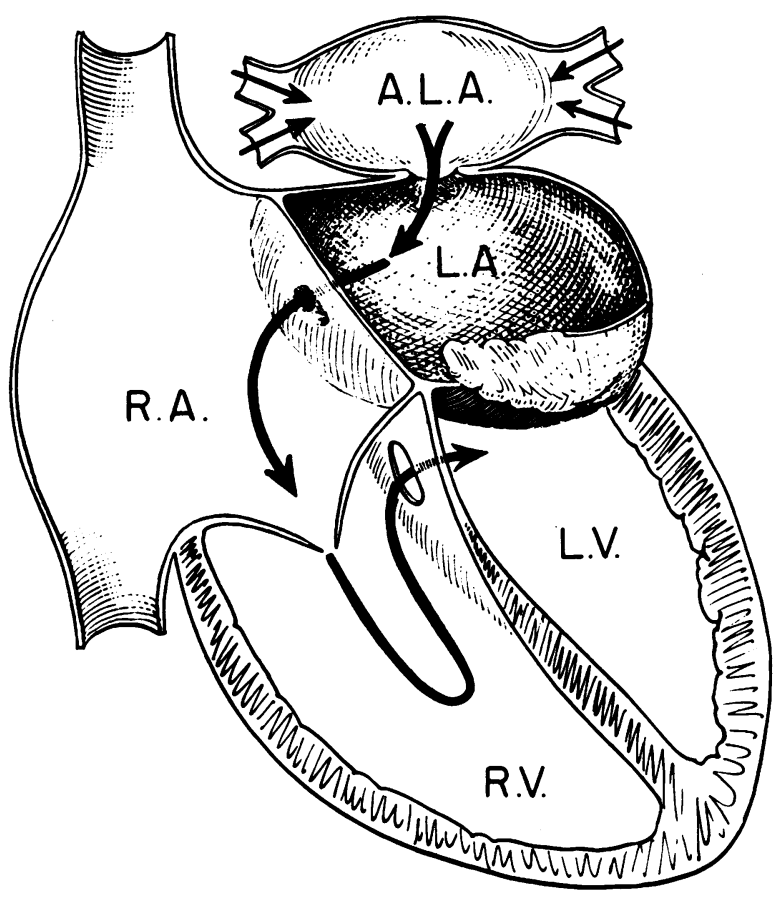

Fig. 10.-Diagrammatic representation of the anomalies in Case 4. The four individual pulmonary veins (short arrows) enter the accessory left atrial chamber (A.L.A.) which communicates through an orifice in its floor with the true left atrium (L.A.). The true left atrium communicates with the left atrial appendage and, through a patent foramen ovale, with the right atrium (R.A.). The right ventricle (R.V.) communicates with the left ventricle (L.V.) through a ventricular septal defect.

and as their lobes develop, there is a stong tendency for the blood supply of each unit of lung to become independent of the supply to the other units (Edwards, 1960). In rare situations, at times with a recognized stimulus, vascular connexions between pulmonary units may persist while the parenchymal elements become separate. This explains the persistence of the venous connexions between the lower and upper lobes of the left lung observed in Case 1 and Case 3, and for the assumed connexions between the upper and lower lobes of the right lung and those between the lower and upper lobes of the left lung in Case 2.

In Cases 1 and 2 the stimulus to persistence of these primitive interlobar venous connexions was afforded by the coexisting mitral atresia and premature closure of the foramen ovale. It is presumed that in Case 3 the persistence of the primitive interlobar venous connexions was necessitated by failure of the pulmonary veins to connect with the atrial portion of the heart. Whatever the stimulus, it must have been present at an early stage when differentiation of the pulmonary lobes was not yet complete and while primitive connexions between the pulmonary venous systems and cardinal veins still existed (Edwards, 1953).

The anomalously connecting veins observed in Cases 1 and 3, in the case of Lucas, and assumed in Case 2, all represented persistence of primitive connexions between the pulmonary and the cardinal venous systems. Each of these anomalous channels served the function of a collateral vessel (Lucas et al., 1962).

Pulmonary venous obstruction occurred in each of the cases here reported. In Cases 1 and 2 and in Lucas' case, this resulted partly or wholly from the coexisting premature closure of the foramen ovale and mitral atresia. It was augmented in Case 1 by compression upon the anomalously connecting veins of contiguous structures, and in Case 2 by much hypoplasia of the right upper pulmonary vein. In Case 3 the pulmonary veins did not connect with the left atrium. Pulmonary 
venous obstruction in this case resulted from compression by adjacent mediastinal structures of two anomalously connecting pulmonary veins. In Case 4, obstruction to pulmonary venous flow was caused by the narrow patency of the foramen ovale in the presence of mitral atresia.

These cases serve to emphasize that the clinical identification of mitral atresia should suggest the possibility of coexistent pulmonary venous anomalies (which themselves may be the site of pulmonary venous obstruction). Conversely, the angiocardiographic demonstration of pulmonary venous anomalies may indicate coexistent mitral atresia.

\section{SUMMARY}

Pulmonary venous anomalies were observed in 5 among 29 specimens with mitral atresia. In 4 of these 5 the anomalous pulmonary veins took the form of anomalous pulmonary venous connexion. In the remaining case the pulmonary venous anomaly was represented by cor triatriatum. In 3 (Group I), mitral atresia and premature closure of the foramen ovale coexisted. The anomalous pulmonary venous connexions in this group provided collateral routes for the flow of pulmonary venous blood, and can be understood as developing in response to obstruction at the foramen ovale when the mitral valve is atretic. In the remaining 2 (Group II) no such causative factor could be invoked. In one of the latter group total anomalous pulmonary venous connexion coexisted with a common atrium. In the other, cor triatriatum coexisted with a patent foramen ovale.

Pulmonary venous obstruction occurred in each of the 5 cases.

\section{REFERENCES}

Becu, L. M., Tauxe, W. N., DuShane, J. W., and Edwards, J. E. (1955). Anomalous connection of pulmonary veins with normal pulmonary venous drainage. Report of case associated with pulmonary venous stenosis and cor triatriatum. Arch. Path., 59, 463.

Burroughs, J. T., and Edwards, J. E. (1960). Total anomalous pulmonary venous connection. Amer. Heart J., 59, 913.

Edwards, J. E. (1953). Pathologic and developmental considerations in anomalous pulmonary venous connection. Proc. Mayo Clin., 28, 441.

(1960). Congenital stenosis of pulmonary veins. Pathologic and developmental considerations. Lab. Invest., 9, 46.

— , and DuShane, J. W. (1950). Thoracic venous anomalies. I. Vascular connection of the left atrium and the left innominate vein (levoatriocardinal vein) associated with mitral atresia and premature closure of the foramen ovale (Case 1). II. Pulmonary veins draining wholly into the ductus venosus (Case 2). Arch. Path., $49,517$.

Elliott, L. P., and Edwards, J. E. (1962). The problem of pulmonary venous obstruction in total anomalous pulmonary venous connection to the left innominate vein. Circulation, 25, 913.

Kauffman, S. L., Ores, C. N., and Andersen, D. H. (1962). Two cases of total anomalous pulmonary venous return of the supracardiac type with stenosis simulating infradiaphragmatic drainage. Circulation, 25, 376.

Lucas, R. V., Jr., Lester, R. G., Lillehei, C. W., and Edwards, J. E. (1962). Mitral atresia with levoatriocardinal vein. A form of congenital pulmonary venous obstruction. Amer. J. Cardiol., 9, 607. 